\title{
Harvesting the Swine Genome: A Roadmap for Quantitative Trait Loci (QTL) Analysis
}

\author{
Jonathan E. Beever and Lawrence B. Schook \\ Department of Animal Sciences \\ Institute for Genomic Biology \\ University of Illinois, Urbana, IL 61801
}

\begin{abstract}
Completion of the human genome sequence provides a foundation for understanding genetic complexity and how it contributes to diverse phenotypes and disease. It is clear that model organisms will continue to play an invaluable role in the synthesis of this understanding. The pig represents an evolutionary clade distinct from primates or rodents and thus, provides considerable power in the analysis of DNA sequence and phenotypic diversity. The pig, a domesticated eutherian mammal, has coevolved with humans and represents a taxon with diverse selected phenotypes. The pig has played a central role in the scientific and medical communities, thus providing scientific justification for understanding the porcine genome with respect to physiological models of growth and development, health, and reproduction. The recent ability to genetically modify the porcine genome, genetically manipulate embryonic fibroblasts, and 'clone' genetically modified somatic cells through nuclear transfer attests to how the pig can provide relevant genetic models of appropriate phenotypes and to resolve the genetic complexity of quantitative traits.
\end{abstract}

\section{INTRODUCTION}

The Porcine Genome. The pig genome is of similar size $\left(3 \times 10^{9} \mathrm{bp}\right)$, complexity, and chromosomal organization $(2 n=38$, including meta- and 
acrocentric chromosomes) as the human genome. Over the past decade tremendous progress has been made mapping and characterizing the swine genome. Currently, moderate to high resolution genetic linkage maps containing highly polymorphic loci (Type II) have been produced using independent mapping populations (Archibald et al., 1995; Ellegren et al., 1994; Rohrer et al., 1996). Additionally, physical mapping methods such as somatic cell hybrid analysis (Rettenberger et al., 1994; Yerle et al., 1996), in situ hybridization, and ZOO-FISH (Chowdhary et al., 1996; Fronicke et al., 1996) have been employed to enrich the Type I marker map, and to perform comparative analysis with map-rich species such as the human and mouse. To date, $>5,000$ mapped loci are cataloged for the pig genome (http://www.thearkdb.org). Recently, whole-genome radiation hybrid (WG-RH) panels (7,500 and 12,500 rad) have been generated for swine (Hawken et al. 1999; Yerle et al. 2002) resulting in yet another rapid increase in the number of expressed sequences being mapped, facilitating comparative mapping with other species (Rink et al. 2002). The swine genomics community has also acquired access to resources such as bacterial artificial chromosome (BAC) libraries (Fahrenkrug et al., 2001; Anderson et al., 2000) that provide approximately 35X coverage of the swine genome. These BAC resources have facilitated the production of high-resolution physical maps in specific chromosomal regions (Milan et al., 2000; Rogel-Gaillard et al., 1999) and support the construction of sequence-ready mapping resources for the porcine genome.

Comparative maps have indicated that the porcine and human genomes are more similarly organized than when either is compared to the mouse (Thomas et al., 2003). The mean length of conserved syntenic segments between human and pig is approximately twice as long as the average length of conserved syntenic segments between human and mouse (Ellergren et al., 1994; Rettenberger et al., 1995). Furthermore, the 
organizational similarities between the human and porcine genomes are reflected in similarities at the nucleotide level. In more than 600 comparisons of non-coding DNAs aligned by orthologous exonic sequences on human chromosome 7, pig (and cow, cat and dog) sequences consistently grouped closer to human and non-human primate sequences than did rodent (mouse and rat) sequences (Thomas et al., 2003). Furthermore, the rodent genomes are evolving at a different (faster) rate than other representative genomes.

Harvesting Genomic Information. The porcine research community has a long history in quantitative genetics, and more recently in genomics research. The genetic contribution of many traits in pigs is well documented, and this knowledge has provided the basis for the identification and mapping of a growing number of QTL (Andersson et al. 1994; Milan et al. 2000; Rohrer et al. 1999; Wilkie et al. 2000; Paszek et al. 2000; Malek et al., 2001a,b; Nezer et al., 2002). The maps have been used to identify chromosomal regions that influence quantitative traits affecting growth, body composition, reproduction and immune response (reviewed by Bidanel and Rothschild, 2002). The QTL regions defined in these studies often span 20-40 centiMorgans (CM) and perhaps correspond to about 20-40 megabase pairs (Mbp) of DNA. These initial scans for the gene(s) controlling the phenotype of interest generally only reduce the search space to $1-2 \%$ of the genome, perhaps to $200-400$ positional candidate genes. Locating the gene(s) responsible and identifying the causal molecular genetic variation is a major challenge.

Many populations have been used to map genes to large chromosomal regions but positional mapping of causal genes has been difficult. The only limitation to performing direct genetic experiments and identifying genes underlying these traits is the lack of a complete genome 
sequence. Sequencing the porcine genome and the generation of a panel of thousands of single nucleotide polymorphisms (SNPs) will provide additional polymorphic markers and positional candidate genes based on the human and mouse genome sequences. Large populations with designed matings can be used to positionally map genes. The populations can be generated by natural reproduction, artificial insemination or assisted reproductive technologies. Clones can also be generated from fibroblasts, or stem cells and cryopreserved. This technology provides the opportunity for knock-out or knock-in experiments in an animal other than the mouse. Interspecies porcine hybrids are easily produced and are very valuable for knockout/in experiments and studying genomic imprinting (Andersson et al., 1994). Thus, comprehensive clone-based physical maps and ultimately a complete genome sequence will facilitate and accelerate the identification of trait genes and the underlying genetic variation.

Justification for Genomic Sequencing. A USDA workshop during the summer of 2002 The Allerton III Conference ("Beyond Livestock Genomics") was designed to bring together leading investigators from broad disciplines (physiology, reproduction, animal health, nutrition, and genetics) to begin to develop a plan for full utilization of genomic information to promote animal health and production. In February 2002, the National Academy of Sciences organized a public workshop, "Exploring Horizons for Domestic Animal Genomics" (NRC, 2002) to identify research goals and funding needs. Subsequent discussions identified a growing need to have a broader context for discussion to ensure full utilization of the genomic information and tools in support of animal research. Thus, the Allerton III Conference provided a venue for discussion of how genome sequences could be harvested to support the broader animal agricultural community, while contributing to life science discovery. The objectives of the Allerton III Conference, included: 1) identification of genomic and 
bioinformatic tools and reagents required to exploit information from the human genome initiative, 2) discussion of needs and opportunities for full implementation of genomic capabilities by related disciplines, and 3) identification of needs and opportunities to ensure full technology transfer and commercialization (Hamernik et al., 2003).

There is significant support for sequencing the porcine genome among the international scientific community. By the end of 2004, the Danish-Chinese Swine Genome Collaboration will be submitting 3,000,000 genomic reads into the public database from a whole-genome shotgun sequencing effort. The Danish-Chinese Swine Genome Collaboration will also submit an additional 1,000,000 EST sequences. A Porcine BAC Consortium with global representation of government, university and industrial constituents has been established to produce a whole-genome porcine BAC map. The non-profit Alliance for Animal Genome Research has provided support for development of a NIH "white paper" (www.genome.gov/10002154) and has provided leadership for convening the National Research Council workshop supported by the USDA, NIH, DOE, and NSF to further define scientific objectives related to this and other sequencing initiatives.

Understanding the Evolutionary Process. While all the eutherian mammalian orders probably diverged $70-80$ million years ago, it is evident that some have genomes that are much more highly conserved relative to primates than others. These domestic animal groups are also more conserved relative to humans than rodents with respect to total genomic structure as revealed by comparative gene mapping and sequence divergence. They themselves are diverse taxa, and over the next few years, each genome should be sequenced to reveal its evolutionary history and to facilitate the important role each animal plays in comparative medicine. 
The domestic pig is somewhat unique, however, in that it represents all the artiodactyls, a phenotypically diverse clade of animals with circumglobal distribution and a conserved number of chromosomal arms. The pig is also unique in that the Wild Boar, Sus scrofa from which the domesticated Sus scrofa was developed, is still present and has a distinct phenotype and different karyotype.

The Swine Genome Sequencing Consortium (SGSC). In September, 2003, interested researchers convened in Jouy-en-Josas, France to establish the SGSC for facilitation and coordination of international efforts toward obtaining the complete porcine genome sequence. A coordinated international effort was initiated to develop a porcine BAC map with two BAC libraries (RPCl-44 and $\mathrm{CHORI}-242$ ) made by Dr. Pieter J. de Jong (CHORI, Oakland, CA, USA), one library made at the Roslin Institute (Anderson et al., 2001), and a library produced at INRA (Robel-Gaillard et al., 1999). The USDA-ARS Meat Animal Research Center, the Roslin Institute, the Sanger Institute, INRA (Jouy-en-Josas and Toulouse) and the University of Illinois are the current participants. USDA-ARS and the Biotechnology and Biological Sciences Research Council have funded the Wellcome Trust Sanger Institute to fingerprint a 5X library developed by Roslin Institute and the 10X CHORI-242 library. In coordination with this effort, the University of Illinois has fingerprinted $5 \mathrm{X}$ of the RPCl-44 library and has sequenced 100,000 BAC-ends. Additionally, INRA has completed the fingerprinting of their $5 \mathrm{X}$ library. Through the exchange of BAC clones, data have been merged to permit a comprehensive analysis. INRA has screened more than 1,000 BACs from this library for known genes and markers and has mapped them on genetic and RH maps. INRA has shared this set of BACs to facilitate anchoring of contigs. Sequencing the ends of all fingerprinted BAC clones has also been conducted. The final product will represent $20 \mathrm{X}$ coverage of the porcine genome. The Sanger 
Institute will maintain a database to store fingerprint, sequence and STS mapping information.

Strategy for Genomic Sequencing. The merits of strategy that we espouse for sequencing the pig genome have been demonstrated by the sequencing team at Baylor College of Medicine Human Genome Center for the rat (Rat Genome Sequencing Project Consortium, 2004). Briefly, the strategy combines the whole genome shotgun (WGS) approach with skim sequencing of BAC clones selected to represent a minimum tiling path (MTP) through the pig genome. We propose that a draft sequence of the pig genome with $6-7 x$ genome coverage be produced by this hybrid approach.

One of the key strengths of the hybrid approach is that the resources (BAC clones) are in place for targeted sequence closure in regions of interest. An important difference between the application of this approach to the pig genome and its use for other species to date is that the porcine fingerprint map and $B A C$ end sequence information will be completed before the sequencing project starts. Thus, it should be possible to determine a BAC tiling path from these two datasets, identifying a set of BACs with minimal overlap at the outset of the sequencing project. Current calculations predict that at most 25,000 BACs will need to be sequence skimmed since the human is approximately $2.9 \mathrm{~GB}$ and mouse and rat about $2.5 \mathrm{~GB}$. This calculation is also supported by the increased size of the BAC inserts from $150 \mathrm{~kb}$ to a range of $160-180 \mathrm{~kb}$, thus reducing the number of BACs to be sequence skimmed. The pig project can then be conducted as for the rat: about 25,000 BAC clones will be sequenced to about $1-2 x$ coverage and the remaining $4-5 x$ coverage will come from WGS sequencing of $3 \mathrm{~kb}, 12 \mathrm{~kb}$, and $35 \mathrm{~kb}$ libraries. It should be possible to reduce the number of BAC DNA preparations and shotgun libraries by 
using the pooled clone array approach, but this may not be necessary as there will be a pre-existing tiling path.

The Sequencing Template. The majority of clones that have been fingerprinted and end-sequenced have come from the CHORI-242 BAC library. This library was constructed from a single female pig that was raised at the University of Illinois. To facilitate sequence assembly, efforts will be made to select as many $\mathrm{CHORI}-242$ clones as possible for the BAC minimum tiling path. Additionally, the WGS libraries will be made from autologous DNA to further enhance sequence assembly between WGS reads and those from the BAC skim. Full-length cDNA libraries will also be constructed from tissues belonging to the original sow or her clones, providing autologous sequence for gene annotation.

Anchoring the Pig Physical Map. In January 2002, a USDA/NRICGP funded project aimed at defining evolutionary breakpoints and internal rearrangements in gene order between the human and porcine genomes was undertaken. Since that time, we have added 2,276 new loci to the porcine $\mathrm{RH}$ map, excluding the $\mathrm{X}$ chromosome. These loci are derived from BAC-end sequences (BESs) generated from the RPCI-44 and CHORI242 porcine BAC libraries including BAC clones that were fingerprinted for construction of the pig genome physical map. Each arm of the metacentric (SSC1-12) and the acrocentric (SSC13-18) pig chromosomes are represented by a single linkage group. The average spacing between loci is $1.3 \mathrm{Mb}$ relative to the human genome sequence. Segments of conserved gene order range from $<0.5 \mathrm{Mb}$ to $75.8 \mathrm{Mb}$ in size with 141 comparative segments being defined by at least 2 loci. These data demonstrate that the scaffold for assembly of the porcine physical map is complete. This resource will be invaluable for the assembly of the BAC minimum tiling path along each of the pig chromosomes. 
Mapping of QTL by Linkage Disequilibrium (LD) Analysis. Historically two methdods have been utilized to map QTL. These have included linkage analysis and LD. Most QTL studies using families derived from divergent crosses and low-resolution maps have used linkage analysis to localize specific traits to chromosomal regions of $<10 \mathrm{cM}$ (Bidanel and Rothschild, 2002). LD in contrast is highly informative in high-resolution fine mapping and can incorporate both population and family data. The development of high-density SNP maps provides a powerful approach for multipoint fine mapping. Recently Fan and Xiong (2002) have shown that a regression approach to mapping QTL by LD using population data can be achieved with a complex trait (disease in their example) of heritability $h^{2} \geq$ 0.15 with a sample size of 250 individuals with moderate LD.

In the analysis of complex diseases in humans, access to large families or informative populations is in most cases rate limiting. Thus, the application of genome-wide association studies using SNPs is more effective (Nowotny et al., 2001). This approach is based on the premise that a SNP may be in LD with the 'true' functional variant. LD also is referred to as "allelic association", which is when alleles at two distinct locations in the genome are more highly associated than expected. To this end, the development of SNP-based LD maps could facilitate wholegenome association studies, leading to more efficient detection of candidate susceptibility genes (or in our case, genes controlling economically important traits) (Nowotny et al., 2001).

Although, the extent of LD is swine has not been determined, several studies in different human populations have been explored and are relevant for these discussions (Collins et al. 2001). Using isolated Finnish and Sardinian populations, LD extended from 427-893 kb (as compared to a large heterogenous population represented by $\mathrm{CEPH}$ ). These results suggest that isolated populations are not essential for LD mapping of traits (Collins et al. 2001). This study also revealed that genome regions with 
extensive and less extensive disequilibrium, when plotted against the physical map, corresponded to recombination hot and cold spots. Other studies have demonstrated that population differences and marker location within the gene may be important factors in the selection of SNPs for use in the study of complex disease (traits) with linkage or association mapping methods (Goddard et al. 2000).

\section{Utilization of Diverse Germplasm to Map Highly Selected Traits.} During the past decade, significant resources have been focused on establishing appropriate divergent crosses of pigs to map QTL. The global approach to mapping specific phenotypes in pigs has utilized divergent crosses between Western breeds (Yorkshire, Large White) and exotic breeds (Chinese Meishan or Wild Boar) (Bidanal and Rothschild 2002). These breeds have had extensive selection pressure for growth, carcass or reproductive traits. Of specific interest are the high reproductive characteristics of the Meishan breed. Imported into both Europe and the U.S. during the 1990s, these animals have been used to map traits such as prolificacy, ovulation rates and age of puberty (White et al. 1993). Significant selection for litter size in China has resulted in significant differences in all phenotypes associated with reproduction. However, the ability and cost to measure and study such low heritability traits has been a deterrent to fine mapping studies and the ability to translate map information into commercial populations.

In an attempt to map reproductive QTL, the University of Illinois created a divergent cross between the exotic Meishan and domestic Yorkshire (Schook and Wheeler, 1995). The parental population was studied extensively (White et al. 1993) in order to maximize phenotypic variation. The resource population (created with 3 Meishan boars and 7 Yorkshire sows resulting in a F2 population of 304 individuals) permitted the mapping eight reproductive traits including: gestation length, number of corpora lutea, total fetuses, uterine length, total piplets born alive, number of stillborn piglets and number of piglets weaned. Of particular interest was 
that the number of corpus luteum [ovulation rate], gestation length, uterine length and stillborn piggets mapped to SSC8 (Wilkie et al., 1999). Genome-wide significance for the number of corpora lutea was observed on SSC8 at position $101 \mathrm{cM}$ [defined by MS S0086 and SW1037] that accounted for $13.2 \%$ of the F2 phenotypic variance. A second SSC8 region was also identified between SW1345 and SW1037 that may reflect a second QTL or lack of informative marker saturation. Subsequently, the presence of a QTL(s) on SSC8 affecting reproduction has been validated using different crosses (Jiang et al., 2001; Rohrer et al., 1999). Further confirmation of a CL located on SSC8 was provided through Rathje et al. (1997). They observed investigating an F2 population produced by crossing a line previously selected for 10 generations by using an index of ovulation rate and embryonic survival and a line selected at random. This study showed strong evidence for a QTL affecting ovulation rate near the telomeric arm of SSC8. Although their conclusion mapped the CL controlling region to a different location on SSC8, the status of the linkage map contributed to this finding since SW205 was located in the centromeric region. Thus, a number of independent studies have confirmed the presence of a QTL on SSC8 that contributes to both female and male phenotypes.

In an effort to further resolve the SSC8 reproductive QTL map, additional efforts have focused on developing higher resolution map of SSC8 in the putative QTL containing region defined by SW205, SW444, SW206 and SW29. Brauschweig et al. (2001), utilizing the UIUC Meishan $x$ Yorkshire resource family generated a dense genetic map in the chromosomal region affecting corpora lutea (CL). Using a swine YAC library and targeted screening, additional microsatellites (MS) were isolated to further enhance the resolution of the QTL containing chromosomal region. MS were mapped using the IMpRH and genotyped on the UIUC resource population. The $C L$ phenotypic data of 108 females the additional 29 markers were used in a least squares regression method. The calculated QTL effect for CL obtained by the multilocus least squares 
method showed a maximum test statistic $(F$ value $=13.98)$ at position $99 \mathrm{cM}$ between three MS derived from the porcine YACs containing SW205 and SW1843 spanning an interval of $7.1 \mathrm{cM}$. The corresponding genome-wide $P$-value was 0.009 . The additive QTL effect explained $17.4 \%$ of the phenotypic variance. Thus, creating a higher resolution map in this region did increase the level of phenotypic variance.

Comparative Mapping by Annotation and Sequence Similarity (COMPASS) of SSC8. BAC-end sequences can be tested for similarity to human and mouse genome sequences by batch BLASTN analysis against finished and draft human (NCBI) and mouse (NCBI) genome sequence. A cutoff value of $e^{-5}$ will be used to identify putative regions of genomic identity. Putative orthologs can then be predicted for their map location on the basis of available comparative mapping information (Band et al., 2000). A single-sequence version has been made available to the public at: http://keck1.biotec.uiuc.edu. We will use the pipeline version that permits batch processing of thousands of sequences simultaneously.

On the basis of our preliminary analysis approximately $25 \%$ of all swine BAC ends had a significant match $\left(e^{-5}\right)$ to a human genome sequence. Thus, on the basis of $80 \%$ sequencing success rate, we expect to obtain 1,000 BACs $\times 0.8 \times .25=200$ new comparatively-anchored sequence tagged sites (CASTS) for SSC8. It has been previously shown with ESTs that this empirically chosen value $\left(e^{-5}\right)$ identifies orthologous human sequences with $>95 \%$ accuracy as determined by parallel radiation hybrid mapping (Band et al., 2001). We recently obtained $100 \%$ success of map prediction with $20 \mathrm{RH}$-mapped BAC ends using the basic COMPASS procedure. These 200 CASTS, randomly distributed over the fingerprintbased contig for SSC8, will enable the construction of a virtual highresolution comparative physical map of the swine and human genomes. Importantly, BAC-ends that fall into regions of uncertainty in the comparative map can then be used to close these "gaps" thus obtaining a higher resolution comparative map. The resulting scaffold will permit 
identification of orthologous segments of the swine, human and mouse genomes thus facilitating the rapid identification of candidate genes and targeted regions for complete DNA sequencing.

Orthologous DNA Sequence Mining: Radiation Hybrid (RH) Mapping. Various cytogenetic approaches have shown that most of HSA4 is homologous with SSC8. Recent utilization of human draft genomic sequences to develop PCR-based genotyping of porcine whole genome radiation hybrid cell lines has resulted in the development of high-resolution comparative maps of expressed sequences. Using the human draft genomic sequence, Jiang, et al. (2002), located 46 orthologs from HSA4 to SSC8. They were able to split SSC8 into nine homologous blocks or conserved segments. They also calculated using a pig-mouse radiation hybrid panel that a ratio of $1 \mathrm{~cm} / 5.2 \mathrm{cRad}$. Thus, their Linkage Group 2 spanned 124 cRad.

Rink et al. (2002) have constructed a comparative porcine/human map that provides $98.3 \%$ coverage of the human genome. However, this map still has over 60 breakpoints and 90 microrearrangments. With respect to SSC8, they mapped only 25 transcripts representing 12 genes, 7 ESTs, and 6 novel ESTs. Thus, their results also demonstrated that SSC8 displayed a low DNA/marker ratio and are "genomic deserts" with respect to mapped transcripts. Thus, additional mining of the human genomic sequence targeted to SSC8 is still required.

Single Nucleotide Polymorphism (SNP) Discovery. Haplotype-based methods offer a powerful approach to mapping polygenic traits based on the association between causal mutations and the ancestral haplotypes on which they arose (Gabriel et al., 2002). As part of the SNP Consortium Allele Frequency Project (human), Gabriel et al. (2002) has characterized haplotype patterns across 51 autosomal regions [13 megabases] in samples from Africa, Europe and Asia. They were able to demonstrate sizeable haplotype blocks where there is little evidence for recombination 
and where only a few common haplotypes are observed with the boundaries and specific haplotypes highly correlated across populations. Relevant to this proposal is their demonstration that such haplotype frameworks provide substantial statistical power in association studies of common genetic variation across each region. These results clearly support our premise for constructing haplotype maps in regions demonstrated to contain QTL.

\section{CONCLUSIONS AND IMPLICATIONS}

Over the next 100 years, world demand for high quality, safe, and nutritious animal products, such as pork, will continue to grow. To keep up with this demand, animal producers will need to harness every resource available to maintain or increase the production of a quality product, while at the same time increasing their competitiveness and profitability in the world market. One of these resources is the "genomic promise;" the promise that complete understanding of all genes and their functions will provide sound answers that can be used to shape the future of animal agriculture.

The investment in animal genomics over the past decade has been significant. However, we are far from fully achieving our goals or exploiting the progress that we have made. The development and use of genomic tools such as genetic, comparative, and physical maps will enable the identification of resources for targeted marker development as well as positional cloning of candidate genes. Furthermore, obtaining the complete genome sequence will lead to the eventual identification and understanding of the genes influencing complex traits in swine. Ultimately, understanding of the detailed genetic mechanisms underlying economically important traits will enhance the competitiveness and profitability of the pork industry. 


\section{ACKNOWLEGEMENTS}

This work was partially supported by grants from the USDA-National Research Initiative (2002-35205-12712), the USDA Cooperative State Research Service (AG2002-34480-11828), the USDA Agricultural Research Service (Agreement No. 58-5438-2-313. The authors also wish to recognize the contributions and assistance of $L$. Liu, and $P$. Schweitzer. A special acknowledgment is made to our colleague $H$. Lewin who has served to stimulate our thoughts and invigorate our pursuits.

\section{REFERENCES}

Anderson, S.I., N.L. Lopez-Corrales, B. Gorick and A.L. Archibald. 2000. A large fragment porcine genomic library resource in a BAC vector. Mammalian Genome 11: 811-814.

Andersson, L., C.S. Haley, H. Ellegren, S.A. Knott, M. Johansson, K. Andersson, L. Andersson-Eklund, I. Edfors-Lilja, M. Fredholm, I. Hansson, J. Håkansson, K. Lundström. 1994. Genetic mapping of quantitative trait loci for growth and fatness in pigs. Science 263:17711774.

Archibald, A.L., C.S. Haley, J.F. Brown, S. Couperwhite, H.A. McQueen, D. Nicholson, et al. 1995. The PiGMaP Consortium linkage map of the pig (Sus scrofa). Mammalian Genome 6:157-175.

Band, M. R., Larson, J.H., Rebeiz, M., Green, C.A., Heyen, D.W., Donovan, J., Windish, R., Stiening, C., Mahyuddin, P., Womack, J.E. and H. A. Lewin. 2000. An ordered comparative map of the cattle and human genomes. Genome Res. 10:1356-1368.

Band, M.R., Olmstead C., Everts, R.E., Liu, Z.L., and Lewin, H.A. 2002. A 3800 gene microarray for cattle functional genomics: comparison of gene expression in spleen, placenta and brain. Animal Biotechnology 13:163-172. 
Bidanel, J. P. and M.F. Rothschild. 2002. Current status of quantitative trait loci mapping in pigs. Pig News and Information 23:N39-N54.

Braunschweig, M.H., A.A. Paszek, J.I. Weller, Y. Da, R.J. Hawken, M.B. Wheeler, L.B. Schook and L.J. Alexander. 2001. Generation and exploration of a dense genetic map in a region of a QTL affecting corpora lutea in a Meishan $x$ Yorkshire cross. Mammalian Genome 12:719-723.

Chowdhary, B.P., Raudsepp, T., Fronicke, L., and H. Scherthan. 1998. Emerging patterns of comparative genome organization in some mammalian species as revealed by Zoo-FISH. Genome Res. 8:577-589.

Collins, A. S. Ennis, P. Taillon-Miller, P.-Y. Kwok and N.E. Norton. 2001. Allelic association with SNPs: metrics, populations, and the linkage disequilibrium map. Human Mutation 17:255-262.

Ellergren, H., B.P. Chowdhary, M. Johansson, L. Marklund, M. Fredholm, I. Gustavsson and L. Andersson. 1994. A primary linkage map of the porcine genome reveals a low rate of genetic recombination. Genetics 137:1089-1100.

Engler, F.W., Hatfield, J., Nelson, W. and Soderlund C.A., 2003. Locating sequence on FPC maps and selecting a minimal tiling path. Genome Research 13: 2152-63.

Fahrenkrug, S.C., G.A. Rohrer, B.A. Freking, T.P.L. Smith, K. Osoegawa, C.L. Shu, J.J. Catanese and P.J. de Jong. 2001. A porcine BAC library with tenfold genome coverage: a resource for physical and genetic map integration. Mammalian Genome 12:472-474.

Fan, R. and M. Xiong. 2002. High resolution mapping of quantitative trait loci by linkage disequilibrium analysis. Eur. J. of Human Genetics 10:607-615.

Fronicke, L., Chowdhary, B.P., Scherthan, H., and I. Gustavsson. 1996. A comparative map of the porcine and human genomes demonstrates 
ZOO-FISH and gene mapping-based chromosomal homologies. Mammalian Genome 7:285-290.

Gabriel, S.B., S.F. Schaffner, H. Nguyen, J.M. Moore et al. 2002. The structure of haplotype blocks in the human genome. Science 296:22252229.

Goddard, K.A.B., P.J. Hopkins, J.M. Hall and J.S. Whitte. 2000. Linkage disequilibrium and allele-frequency distributions for 114 singlenucleotide polymorphisms in five populations. Am. J. Hum. Genet. 66:216-234.

Goddard, K.A.B. and E.M. Wijsman. 2002. Characteristics of genetic markers and maps for cost-effective genome screens using diallelic markers. Genet. Epidemiology 22:205-220.

Gregory, S.G., M. Sekhorn, J. Schein et al. 2002. A physical map of the mouse genome. Nature 418:743-749.

Hamernik, D.L., H.A. Lewin and L.B. Schook. 2003. Allerton III. Beyond Livestock Genomics. Animimal Biotechnology 14:77-82.

Hawken, R.J., J. Murtaugh, G.H. Flickinger, M. Yerle, A. Robic, D. Milan, J. Gellin, C.W. Beattie, L.B. Schook and L.J. Alexander. 1999. A first generation porcine whole-genome radiation hybrid map. Mammalian Genome 10:824-830.

Jiang, X., H.He, N. Hamasima, H. Suzuki and A.M.V. Gibbins. 2002. Comparataive mapping of Homo sapiens chromosome 4 (HSA4) and Sus scrofa chromosome 8 (SSC8) using orthologous genes representing different cytogenetic bands as landmarks. Genome 45:147-156.

Malek, M., J.C.M. Dekkers, H.K. Lee, T.J. Baas, and M.F. Rothschild. 2001a. A molecular genome scan analysis to identify chromosomal region influencing economic traits in the pig. I. Growth and body composition. Mammalian Genome 12:630-636. 
Malek, M., J. C. M. Dekkers, H. K. Lee, T. J. Baas, K. Prusa, E. HuffLonergan, and M. F. Rothschild. 2001b. A molecular genome scan analysis to identify chromosomal regions influencing economic traits in the pig. II. Meat and muscle composition. Mammalian Genome 12:630-636.

Milan, D., T.T. Jeon, C. Looft, V. Amarger, A. Robic, M. Thelander, C. Rogel-Gaillard, S. Paul, N. lannuccelli, L. Rask, H. Ronne, K. Lundstrom, N. Reinsch, J. Gellin, E. Kalm, P. Le Roy, P. Chardon, and L. Andersson. 2000. A mutation in PRKAG3 associated with excess glycogen content in pig skeletal muscle. Science 288:1248-1251.

Mouse Genome Sequencing Consortium. 2002. Initial sequencing and comparative analysis of the mouse genome. Nature 420:520-562.

Nezer, C. L. Moreau, D. Wagenaar and M. Georges. 2002. Results of a whole genome scan targeting QTL for growth and carcass traits in a Pietrain X Large White intercross. Gen. Sel. Evol. 34:371-387.

Nowotney, P., J.M. Kwon and A.M. Goate. 2001. SNP analysis to dissect human traits. Current Opin. in Neurobiol. 11:637-641.

Paszek, A. A., P.J. Wilkie, G.H. Flickinger, G.A. Rohrer, L.J. Alexander, C.W. Beattie, and L.B. Schook. 1999. Interval mapping of growth in divergent swine cross. Mammalian Genome 10:117-22.

Rathje, T.A., G.A. Rohrer and R.K. Johnson. 1997. Evidence for quantitative trait loci affecting ovulation rate in pigs. J. Anim. Sci. 75:1486-1494.

Rat Genome Seqeuncing Project Consortium. 2004. Genome sequence of the Brown Norway rat yields insights into mammalian evolution. Nature 428:493-521.

Rettenberger, G., C. Klett, U. Zecher, J. Kunz, W. Vogel and H. Hameister. 1995. Visualization of the conservation of synteny between humans and pigs by heterologous chromosomal painting. Genomics 26:372-378. 
Rink, A. E.M. Santchi, K.M. Eyer, B. Roelofs, M. Hess, M. Godfrey, E.K. Karajusuf, M. Yerle, D. Milan and C.W. Beattie. 2002. A first-generation EST RH comparative map of the porcine and human genome. Mammalian Genome 13:578-587.

Rohrer, G.A., L.J. Alexander, Z. Hu, T.P.L. Smith, J.W. Keele and C.W. Beattie. 1996. A comprehensive map of the porcine genome. Genome Research 6:371-391.

Rohrer, G.A., J.J. Ford, T.H. Wise, J.L. Vallet and R.K.Christenson. 1999. Identification of quantitative trait loci affecting female reproduction traits in a multigeneration Meishan-White Composite swine population. J. Anim. Sci. 77:1385-1391.

Rohrer, G.A., J.J. Ford, T.H. Wise, J.L. Vallet and R.K.Christenson. 1999. Identification of quantitative trait loci affecting female reproduction traits in a multigeneration Meishan-White Composite swine population. J. Anim. Sci. 77:1385-1391.

Rogel-Gaillard, C., N. Bourgeaux, A. Billaut, M. Vaiman and P. Chardon. 1999. Construction of a swine BAC library: application to the characterization and mapping of porcine type $\mathrm{C}$ endoviral elements. Cytogenet. Cell Genet. 85:205-211.

Schook, L.B. and M. Wheeler. 1994. The University of Illinois Meishan $\mathrm{x}$ Yorkshire swine family. J. Anim. Sci. 7:1920.

Thomas, J.W., J.W. Touchman, R.W. Blakesley, G.G. Bouffard, S.M. Beckstrom-Sternberg, E.H. Margulies, M. Blanchette, A.C. Siepel, P.J. Thomas, J.C. McDowell, B. Maskeri, N.F. Hansen, M.S. Schwartz, R.J. Weber, W.J. Kent, D., et al. 2003. Comparative analyses of multispecies sequences from targeted genomic regions. Nature 424: 788793.

Wilkie, P.J., A.A. Paszek, C.W. Beattie, L.J. Alexander, M.B. Wheeler, L.B. Schook. 1999. A genomic scan of porcine reproductive traits 
reveals a possible quantitative trait loci (QTL) for number of corpora lutea. Mammalian Genome 10:573-8.

White, B.R., D.G. McLaren, P.J. Dziuk and M.B. Wheeler. 1993. Age at puberty, ovulation rate, uterine length, prenatal survival and litter size in Chinese Meishan and Yorkshire females. Theriogenology 40:85-97.

Yerle, M., G. Echard, A. Robic, A. Mairal, C. Dubut-Fontana, J. Riquet, P. Pinton, D. Milan, M. Gillois and J. Gellin. 1996. A somatic cell hybrid panel for pig regional gene mapping characterized by molecular cytogenetics. Cytogenet. Cell Genet. 73:194-202.

Yerle, M., P. Pinton, D. Chantal, A. Nadege, D. Milan and A. Robic. 2002. Generation of a 12,000 rad radiation hybrid panel for fine mapping in pigs. First comparisons between $\mathrm{ImpRH}(7,000 \mathrm{rad})$ and IMNRH2 (12,000 rad). Cytogenet Genome Res. 97:219-228. 